\title{
Rahmenbedingungen für die Bereitschaft junger Schweizer Frauen zur Spende von Eizellen
}

\author{
Über die Zulassung der Eizellenspende wird zurzeit in der Schweiz auf politischer \\ Ebene diskutiert. Mit einer Befragung potentieller Spenderinnen wollten Mediziner \\ des Unversitätsspitals Basel erfahren, ob Frauen zur Eizellenspende prinzipiell bereit \\ wären und wie sie dies begründen.
}

\section{Anna Raggi Nüssli, \\ Gideon Sartorius, \\ Isabelle Bienz, \\ Rebecca Moffat, \\ Christian De Geyter}

Klinik für Gynäkologische Endokrinologie und Reproduktionsmedizin Universitätsspital Basel, Universität Basel
Korrespondenz:

Prof. Dr. med.

Christian De Geyter

Klinik für Gyn. Endokrinologie und Reproduktionsmedizin

Spitalstrasse 21

CH-4031 Basel

Tel. 0612659315

Fax 0612659194

christian.degeyter[at]usb.ch

\section{Einführung}

Gewisse Formen der weiblichen Infertilität können nur mittels Verwendung von Eizellen einer anderen Frau überwunden werden. Im Gegensatz zur Samenspende ist die Spende von Eizellen im Rahmen des Fortpflanzungsmedizingesetzes (FMedG, 1998) in der Schweiz derzeit aber noch verboten. Trotzdem liessen sich im Jahre 2011 mehrere Hundert Schweizer Paare mit unerfülltem Kinderwunsch in ausländischen Zentren behandeln, in denen gespendete Eizellen für reproduktionsmedizinische Behandlungen zur Verfügung stehen. Im Gegensatz zu Therapien in der Schweiz werden Behandlungen im Ausland statistisch nicht erfasst, sodass das tatsächliche Ausmass dieses Reproduktionstourismus nur abgeschätzt werden kann (vermutlich ca. 1000 Behandlungszyklen im Jahre 2011). In einem Urteil des Europäischen Gerichtshofes in Strassburg aus dem Jahre 2010 wurde festgestellt, dass das bisherige Verbot der Eizellenspende bei gleichzeitiger Zulassung der Samenspende in Österreich diskriminierend ist. Am 3.11.2011 hielt die grosse Kammer des Europäischen Gerichtshofes jedoch in nächster Instanz fest, dass die Europäische Menschenrechtskonvention (EMRK) durch das Verbot der Eizellenspende nicht verletzt wird, sodass es nach wie vor im Ermessen der einzelnen Staaten liegt, diese Therapieform zu ermöglichen oder zu untersagen. Dementsprechend muss das bisherige Verbot der Eizellenspende auch in der Schweiz hinterfragt und überdacht werden.

Wieso wurde die Eizellenspende verboten, während die Samenspende vom Gesetzgeber zugelassen wurde? Ursprünglich sollte dadurch die «nicht-natürliche», «gespaltene Mutterschaft» verhindert werden, da vermutet wurde, dass die Trennung von genetischer und biologischer Mutterschaft dem Kindeswohl entgegenstehen kann. Diese Argumentation beruht allerdings nur auf Vermutungen, da es keine gesicherten Erkenntnisse darüber gibt, warum eine Aufspaltung der Mutterschaft für Kinder schädlich sein soll. Zudem ist der Begriff der «Natürlichkeit» in Zeiten der hochtechnisierten Medizin als alleinstehendes Argument relativ inhaltsarm, sodass die Stichhaltigkeit des Verbotes hinterfragt und neu bewertet wer-

\section{Enquête sur l'engagement de donneuses potentielles d'ovocytes en Suisse}

Le débat politique actuel sur le don d'ovocytes en Suisse se concentre uniquement sur l'avantage potentiel des patientes, qui pourraient bénéficier d'un traitement et sur le manque de transparence causé par le tourisme de reproduction. Le motif des donneuses potentielles en Suisse n'a jusqu'à présent guère été considéré. Nous avons fait une enquête parmi des jeunes femmes dans la région bâloise pour mieux connaître leur attitude vers le don d'ovocytes. Nous leurs avons présenté trois scénarios possibles: 1. le don d'ovocytes motivé par l'altruïsme pour aider d'autres femmes affectées par la ménopause précoce; 2 . le don d'ovocytes pour vérifier la qualité d'une parti d'un stock d'ovocytes congelé pour sa propre reproduction plus tard dans la vie; 3 . le don d'une parti de ses ovocytes pour financer son propre traitement avec la procréation médicalement assistée.

Nous avons obtenu des réponses valables de 172 participantes, dont $56,4 \%$ auraient participé à un don d'ovocytes. La motivation la plus fréquente parmi les participantes à notre enquête était le don par altruïsme ( $50 \%$ ), suivi par la congélation d'une partie d'un stock d'ovocytes (46,4\%). En conclusion, notre enquête démontre la volonté de jeunes femmes suisses de la région bâloise de donner des ovocytes à d'autres femmes en occurrence d'un traitement d'infertilité. II importe de préciser que notre enquête est exploratoire puisque la motivation à remplir le questionnaire présuppose une attitude plutôt positive et que la population questionnée n'est pas représentative de l'ensemble des jeunes filles en Suisse. Nous espérons cependant que notre initiative puisse encourager d'autres études systématiques. 
den muss. Als weiteres Argument muss angefügt werden, dass die Spende von Eizellen im Gegensatz zur Samenspende mit einer wesentlich grösseren physischen und psychischen Belastung der Spenderinnen verbunden ist. Die Eizellenspende wird üblicherweise bei jungen Frauen vorgenommen, da die Entwicklungsfähigkeit von Eizellen junger Spenderinnen wesentlich besser und somit die Wahrscheinlichkeit einer erfolgreichen Schwangerschaft höher ist.

Für die Spenderin gibt es keinen unmittelbaren gesundheitlichen Gewinn. Im Gegenteil, die Risiken der ovariellen Stimulationsbehandlung und der Eizellgewinnung mittels transvaginaler Follikelpunktion überwiegen, obwohl sie selten sind. So wurden in 886 Zyklen sechs $(0,7 \%)$ schwerwiegende Komplikationen beobachtet: ovarielles Überstimulationssyndrom (OHSS), Stieldrehung des stimulierten Ovars, Ruptur von Ovarialzysten und Infektionen. Hinzu kamen in 8,6\% der Fälle auch geringfügige Komplikationen, wie mildes OHSS, Harnwegsinfekte oder intra-abdominelle Blutungen, welche ambulant behandelt werden konnten [1]. Durch die Möglichkeit der Ovulationsinduktion mit einem GnRH-Agonisten anstatt humanem Chorion-Gonadotropin (hCG) kann das Risiko eines mittelschweren bis schweren OHSS nahezu gänzlich vermieden werden [2].

\section{Wie werden Spenderinnen rekrutiert?}

Falls auch in der Schweiz die Spende von Eizellen gesetzlich zugelassen werden sollte, stellt sich die Frage, unter welchen Bedingungen man hierzulande Spenderinnen rekrutieren könnte. Wie liessen sich junge Frauen zur Spende ihrer Eizellen motivieren?

In den verschiedenen europäischen und nichteuropäischen Ländern, in denen die Eizellenspende erlaubt ist, gelten jeweils sehr unterschiedliche Richtlinien: In Spanien dürfen die Spenderinnen zwischen 18 und 35 Jahren alt und kinderlos sein. Die Spende ist anonym und die Spenderinnen erhalten zur Aufwandsentschädigung eine bescheidene Geldsumme. In Frankreich ist die Eizellenspende unentgeltlich und ebenfalls anonym, jedoch müssen die Spenderinnen bereits eigene Kinder haben und dürfen nicht älter als 37 Jahre sein. Das Angebot an Spenderinnen ist dementsprechend klein, und die Wartezeiten für Empfängerinnen sind sehr lang. Das von der Europäischen Gesellschaft für Reproduktionsmedizin und Embryologie (ESHRE) empfohlene Alter für Spenderinnen liegt zwischen 24 und 34 Jahren [3].

Für Frauen wurde inzwischen auch eine andere Möglichkeit entwickelt, um die Familienplanung in einem späteren Lebensabschnitt zu realisieren. Heute ist es möglich, mit der Gewinnung und Einfrierung von Eizellen in einem jungen Lebensabschnitt die Fertilitätschancen in einem späteren Lebensabschnitt zu erhöhen. Durch die Entwicklung der Vitrifikation, einer ultraschnellen Form der Kryokonservierung von Eizellen und Embryonen, kann das Entwicklungs-

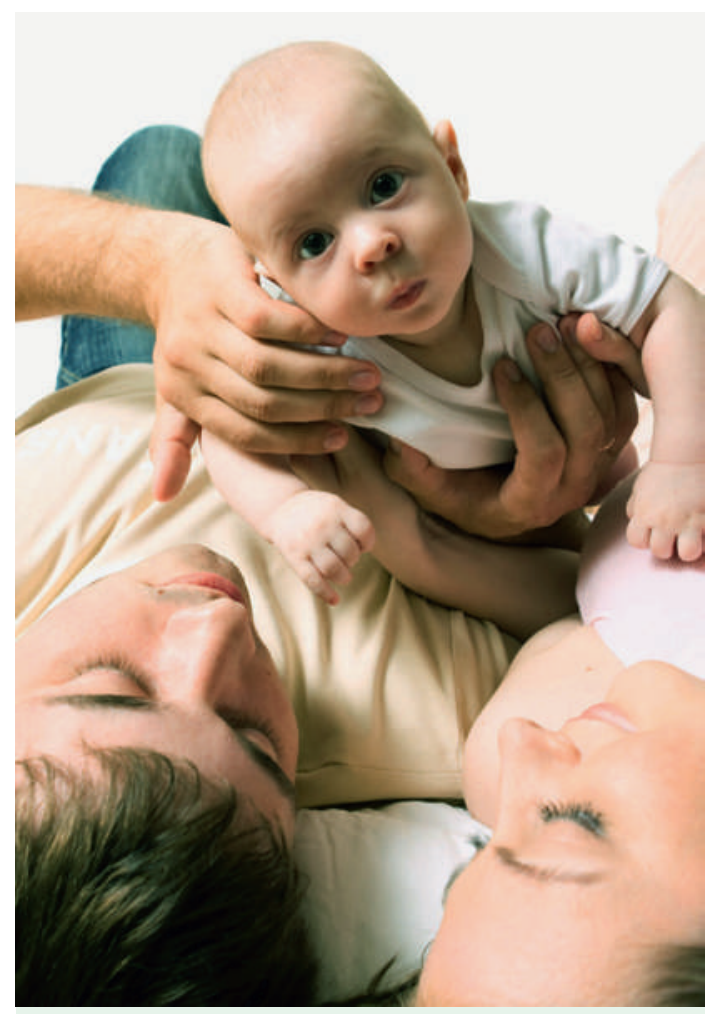

Der Kinderwunsch lässt sich bei manchen Paaren nur mit Hilfe der Reproduktionsmedizin verwirklichen.

potential unbefruchteter Eizellen nahezu unbeeinträchtigt erhalten werden und so eine Fertilitätsreserve angelegt werden. Diese Methode, im englischen Sprachraum auch als «social egg freezing» bezeichnet, wirft jedoch ethische, juristische und finanzielle Fragen auf, die noch ungelöst sind $[4,5]$. Bereits heute werden regelmässig Fertilitätsreserven für Frauen angelegt, die sich aufgrund einer bösartigen Erkrankung einer Chemo- und/oder Radiotherapie unterziehen müssen und dadurch möglicherweise sowohl die endokrine Funktion als auch die reproduktive Funktion der Ovarien verlieren werden [6].

Angesichts dieser neuen technischen Möglichkeiten haben wir drei verschiedene Szenarien aufgestellt, mit denen junge Frauen in der Schweiz möglicherweise dazu motiviert werden könnten, ihre Eizellen zu spenden. In einem Online-Interview wurden junge Schweizerinnen befragt, welches der drei Szenarien am ehesten Anklang findet.

\section{Rekrutierung und Methoden}

Wir haben in der Basler Region junge Schweizer Frauen über 18 Jahre befragt, ob und unter welchen Bedingungen sie bereit wären, Eizellen zu spenden. Drei verschiedene Szenarien für die Spende von Eizellen wurden vorgestellt und bezüglich Akzeptanz und Praktikabilität ausgewertet:

1. «Spende aus Nächstenliebe»: die Spende von Eizellen aus einer rein altruistischen Motivation:

Junge Frauen sind der Meinung, dass eine Eizel- 
lenspende aus Nächstenliebe sinnvoll ist. Spenderinnen werden über einen Zeitraum von circa 12 Tagen mit subkutanen Gonadotropininjektionen zur Stimulation der ovariellen Follikelreifung behandelt. Nach Vervollständigung der Follikelreifung wird die Meiose der Eizellen anstatt mit humanem Choriongonadotropin mit einem GnRH-Agonisten eingeleitet, so dass ein OHSS gänzlich vermieden werden kann. Die Eizellen werden dann im Rahmen einer kurzen Narkose oder leichten Betäubung durch eine transvaginale, ultraschallkontrollierte Punktion der Ovarien gewonnen. Für den entstandenen Aufwand werden die Spenderinnen finanziell entschädigt.

2. «Egg storage»: Die Spenderin möchte aus persönlichem Interesse eine Fertilitätsreserve anlegen, möchte aber die Qualität und Befruchtungsfähigkeit der Eizellen durch die Spende einiger Eizellen überprüfen lassen.

Immer mehr junge, gutausgebildete Frauen mit hohen Karrierezielen möchten sich beruflich etablieren, bevor sie später selbst eine Familie gründen und Kinder bekommen. Sie sind sich bewusst, dass aufgrund des Alters ein erhöhtes Risiko besteht, dass es für die Erfüllung des Kinderwunsches irgendwann zu spät sein könnte. Um dieses Risiko zu vermeiden, möchten sie in jungen Jahren eine hormonelle Stimulation der Ovarien durchführen, Eizellen gewinnen und kryokonservieren lassen und so für sich selber eine Fertilitätsreserve anlegen (im Sinne des «social freezing»). Da es bei dieser Methode jedoch nicht sicher ist, ob die Anzahl und die Qualität der kryokonservierten Eizellen für die eigene Familienplanung ausreichend sind, werden einige dieser Eizellen für eine Spende zur Verfügung gestellt. Im Rahmen dieser Spende kann dann überprüft werden, ob die gewonnenen Eizellen über eine ausreichende Entwicklungsfähigkeit verfügen, um später die Familienplanung der Spenderin zu gewährleisten.

3. «Egg sharing»: Durch die Spende können die Kosten der eigenen Behandlung mit IVF oder ICSI bei Infertilität reduziert werden.

Junge Frauen mit Kinderwunsch planen eine Schwangerschaft. Aufgrund eines Fertilitätsproblems des Partners oder aufgrund einer eileiterbedingten Infertilität wird hierfür eine Behandlung mit ICSI oder IVF benötigt. Da die Kosten einer solchen Therapie in der Schweiz nicht von der Krankenkasse übernommen werden und die Therapie sehr kostspielig ist, beschliesst das Paar einen Teil der für die eigene Behandlung gewonnenen Eizellen zu spenden. Durch diese Spende können die Kosten für die eigene Therapie reduziert werden.

Diese drei Szenarien wurden in einem Fragebogen zusammengefasst und in einfacher Sprache erläutert.

\section{Tabelle 1}

Begründung, weshalb Frauen keine Eizellen spenden möchten ( $n=75$, mehrere Antworten möglich).

Gründe gegen eine Spende

Total

Aus ethischen Gründen

Hormonelle Behandlung unerwünscht

Anderes

Eizellenspende grundsätzlich nicht nötig $4(5,3)$

Der Fragebogen wurde mehrfach von Fachleuten und von Laien korrigiert und auf Verständlichkeit geprüft. Nach der Genehmigung durch die Ethikkommission wurde der Fragebogen auf einer Internetseite aufgeschaltet. Der Link zur Internetseite wurde primär im Umfeld von Mittelschülerinnen verbreitet, wobei später auch verschiedene Freizeitgruppen (Schule, Volleyballverein usw.) angeschrieben und gebeten wurden, den Fragebogen auszufüllen.

Um mehr junge Frauen aus verschiedenen Gesellschaftsgruppen zu erreichen, haben wir mittels Werbekampagne und Inserat in Facebook auf unsere Online-Befragung hingewiesen. Durch das Anklicken eines Links wurden die Interessentinnen zum Fragebogen geleitet. Der Link wurde ebenfalls auf der Internetseite des Universitätsspitals Basel veröffentlicht.

\section{Ergebnisse}

Innerhalb der ersten 8 Wochen erhielten wir 109 Antworten, wovon 102 vollständig waren. Innerhalb der folgenden 12 Wochen wurde die Kampagne auf Facebook lanciert und der Link zur Internetseite wurde 339 Mal angeklickt. Leider resultierten daraus nur ausgefüllte 9 Fragebögen. Über die Internetseite des Basler Universitätsspitals erhielten wir innerhalb von 12 Wochen 61 weitere Antworten.

Insgesamt konnten 172 vollständig ausgefüllte Fragebögen ausgewertet werden.

$78 \%$ der Frauen, die geantwortet haben, waren zwischen 18 und 24 Jahren alt. 22\% waren älter, da-

\section{Abbildung 1}

Grundsätzliche Bereitschaft zur Spende eigener Eizellen $(n=172)$.

\section{Bist Du für eine Eizellenspende bereit?}

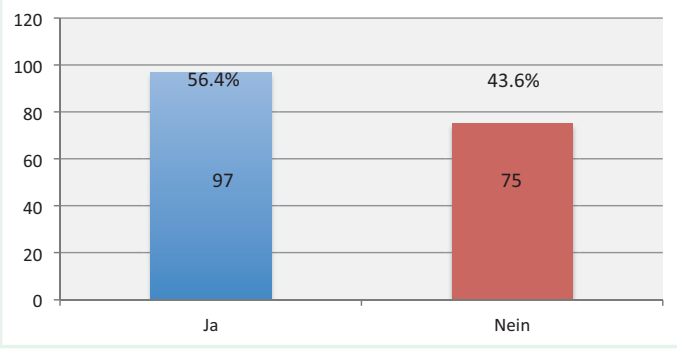


Abbildung 2

Motivation bei spendewilligen Frauen ( $n=97$, mehrere Antworten möglich).

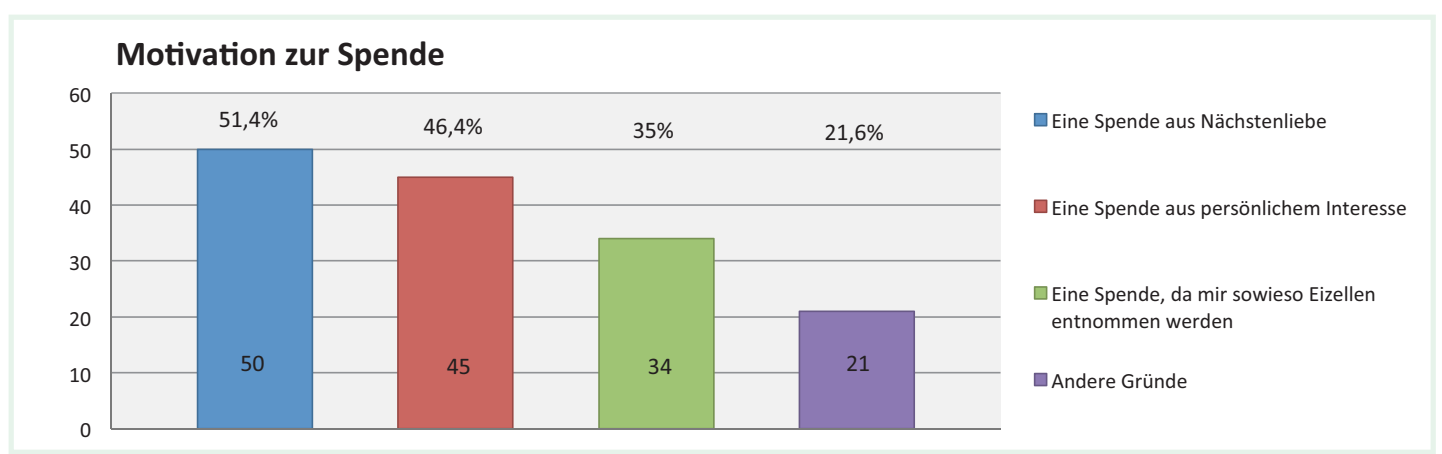

von 6 zwischen 36 und 43 Jahren. 95\% Frauen haben noch keine Kinder, und 40\% dieser jungen Frauen befanden sich in der universitären Ausbildung oder an einer Hochschule.

Insgesamt waren 56,4\% der befragten Frauen zur Spende eigener Eizellen bereit (Abb. 1). Von den drei vorgeschlagenen Szenarien wurde bei $51,6 \%$ der Spende-bereiten Frauen die altruistische Variante favorisiert. Die Variante «egg storage» wurde von $46,4 \%$ der Befragten favorisiert, und 35\% der Befragten würden am «egg sharing» teilnehmen (Abb. 2). Mehrere Antworten waren möglich.

Insgesamt haben sich 43,6\% der Befragten gegen eine Spende von Eizellen ausgesprochen. Die Gründe waren zur Hälfte die Angst vor der hormonellen Therapie, zur anderen Hälfte ethische Aspekte (Tab. 1).

Lediglich 5,3\% aller angefragten Frauen halten eine Eizellenspende grundsätzlich für unnötig.

32\% von 169 Frauen halten eine finanzielle Entschädigung für die Spende von Eizellen für erforderlich: Der als sinnvoll eingeschätzte Betrag variiert allerdings beachtlich und schwankt zwischen 100 Franken und 2500 Franken.

Für mehr als die Hälfte der Befragten sollten nur Frauen bis zum 40. Lebensjahr eine Eizellenspende erhalten dürfen. Zwei Frauen sehen die Altersgrenze bei 50 Jahren, während sechs Frauen die Meinung vertraten, dass eine Eizellenspende unbeschränkt möglich sein sollte.

\section{Diskussion}

Der Wunsch nach einem eigenen Kind ist für sehr viele Paare ein wichtiges, existentielles Bedürfnis. In ca. $10 \%$ der Fälle bleibt dieser Wunsch jedoch unerfüllt. Dank der assistierten Reproduktionsmedizin (ART) kann vielen dieser Paare geholfen werden. Trotz allen Bemühungen bleibt aber $1 / 3$ der behandelten Paare kinderlos.

Ein häufiger Grund dafür ist die altersbedingte Ovarialinsuffizienz der Frau. Im Jahre 2012 lag das mütterliche Durchschnittsalter bei Geburten in der Schweiz bei 31,5 Jahren (Bundesamt für Statistik, 2013).
In der festen Überzeugung, dass Karriere, Partnerschaft und eigene Interessen zunächst wichtiger sind, wird der Zeitpunkt der Gründung einer eigenen Familie oft so weit herausgezögert, bis es zu spät ist. Deswegen hat der Anteil der erfolglos behandelten Frauen in den letzten Jahren zugenommen. Mehr als die Hälfte der Frauen, die im Jahre 2006 eine neue ART-Behandlung begonnen haben, waren zwischen 35 und 44 Jahren alt [7]. Die Fertilität der Frau und die Erfolgschancen einer Sterilitätsbehandlung nehmen jedoch mit 38 Jahren drastisch ab [8]. Viele Frauen mit Kinderwunsch sind sich der Tatsache der abnehmenden Fruchtbarkeit jedoch nicht bewusst und hegen die falsche Hoffnung, dass die Reproduktionsmedizin auch noch in höherem Alter zu Schwangerschaften mit eigenen Eizellen verhelfen kann. Dagegen bleiben die Chancen einer erfolgreichen Therapie bei der Eizellenspende unabhängig vom Alter der Empfängerin erhalten [9].

Für Laien ist es auch irreführend, dass in den Medien immer wieder über Schwangerschaften bei Frauen älter als 45 Jahre berichtet wird. Hierbei wird oft nicht erwähnt, dass diese nur durch Eizellenspende schwanger werden konnten.

Behandlungen unter Verwendung gespendeter Eizellen sind in der Schweiz nicht erlaubt, stellen jedoch für gewisse infertile Frauen eine wichtige, im Ausland etablierte Alternative dar. Dies betrifft Frauen mit einer erschöpften Eizellenreserve, zum Beispiel aufgrund der operativen Entfernung von Ovarien oder aufgrund einer Chemo- oder Radiotherapie des kleinen Beckens oder bei vorzeitiger Menopause oder bei genetischen Erkrankungen, wie dem Ullrich-Turner-Syndrom. Diese Erkrankungen stellten zunächst in den 80er-Jahren die häufigsten Indikationen für eine Spende von Eizellen dar [10]. Später wurden immer mehr Frauen behandelt, die im Zuge des heutigen Trends zur späteren Familienplanung aufgrund einer frühzeitigen Ovarialinsuffizienz nicht mehr schwanger werden konnten [11]. Bei diesem Frauen im fortgeschrittenen Alter zeigen jedoch Schwangerschaften nach Eizellenspende ein erhöhtes Risiko für eine Schwangerschafts-induzierte Hy- 
pertonie und Blutungen unabhängig vom Alter, Parität und Alter der Spenderin und des Partners der Empfängerin [12]. Im Vorfeld einer Behandlung wird daher eine ausführliche Beratung über die psychosozialen Aspekte der Eizellenspende dringend empfohlen.

Derzeit ist die Spende von Eizellen in der Schweiz aufgrund der Richtlinien des Fortpflanzungsmedizingesetzes (FMedG) aus dem Jahre 1998 verboten, sodass sich jährlich ca. tausend Paare zu einer Behandlung ins Ausland begeben. Nicht immer erfolgt die Behandlung dort unter optimalen Bedingungen, wobei besonders häufig das hohe Risiko für Mehrlingsschwangerschaften in Kauf genommen wird. Da derzeit auf politischer Ebene über die Zulassung der Eizellenspende diskutiert wird, haben wir mit dieser Befragungsstudie die Rahmenbedingungen für eine spätere Durchführung einer Eizellenspende evaluiert.

\section{«Viele Frauen mit Kinderwunsch sind sich der Tatsache der abnehmenden Fruchtbarkeit jedoch nicht bewusst.»}

Wir präsentieren hiermit die erste Studie, die sich mit der Eizellenspende und deren Akzeptanz in der Schweiz befasst.

Die Meinungen des befragten Kollektivs junger Schweizer Frauen zeigen eine erstaunlich hohe Bereitschaft junger Schweizerinnen zur Spende von Eizellen. Nur 22\% aller Befragten finden die Eizellenspende aus ethischen Gründen nicht vertretbar. Die meisten würden eine Spende von Eizellen aus rein altruistischen Gründen akzeptieren, und die Mehrheit wünscht keinerlei Entschädigung für eine Spende von Eizellen. Allerdings ist einschränkend anzumerken, dass die Bereitschaft, den Fragebogen auszufüllen, eine gewisse positive Voreingenommenheit der Befragten voraussetzt und die Ergebnisse dieser Befragung nicht repräsentativ für die Gesamtheit junger Frauen in der Schweiz sind. Die Bereitschaft zur Spende von Eizellen scheint aber bei gewissen jungen Schweizerinnen vorhanden zu sein.

Diese Befragung ist die erste dieser Art in der Schweiz und hat überwiegend junge Frauen aus der Nordwestschweiz erreicht. Wir hoffen, dass unsere Studie dazu anregen wird, dass nun aussagekräftigere repräsentative Befragungen unter jungen Frauen in der Schweiz durchgeführt werden. Wir hoffen ebenfalls, mit unserer Initiative zur dringend notwendigen Diskussion zur überfälligen Anpassung des Fort- pflanzungsmedizingesetzes beizutragen [13]. Dieses wurde zu einer Zeit in Kraft gesetzt, als die technischen Voraussetzungen für die erfolgsversprechende Spende von Eizellen noch nicht gegeben waren. Nun ist es aber anders: Die kumulative Schwangerschaftsrate bei der Eizellenspende ist sehr hoch (circa 55\% [9]) und wird vielen Frauen helfen, trotzdem ein Kind zu gebären.

\section{Literatur}

1 Maxwell KN, Cholst IN, Rosenwaks Z. The incidence of both serious and minor complications in young women undergoing oocyte donation. Fertil Steril. 2008;90:2165-71.

2 Youssef MA, Van der Veen F, Al-Inany HG, Griesinger G, Mochtar MH, van Wely M. Gonadotropin-releasing hormone agonist versus hCG for oocyte triggering in antagonist assisted reproductive technology cycles. Cochrane Database Syst Rev. 2010;11:CD008046.

3 Shenfield F, de Mouzon J, Pennings G, Ferraretti AP, Nyboe Andersen A, de Wert G, et al. The ESHRE Taskforce on Cross Border Reproductive Care. Cross border reproductive care in six european countries. Hum Reprod. 2010;25:1361-8.

4 Wunder D. Social freezing in Switzerland and worldwide - a blessing for women? Swiss Med Wkly. 2013;143:w13746.

5 Von Wolf M. «Social freezing»: Sinn oder Unsinn? Schweiz Ärztezeitung. 2013;94(10):393-5.

6 Wunder D, Huober-Zeeb C, Moffat R, Stiller R, Ambrosetti A, Xie M, et al. Schweizerische Empfehlungen zur Fertilitätserhaltung für Patientinnen und Patienten im fertilen Alter vor zytotoxischen Therapien. Schweiz Med Forum. 2012;12:708-9.

7 Bundesamt für Statistik, BFS Aktuell 2007, Bestellnummer: 532-0716-05.

8 Faddy MJ, Gosden RG, Gougeon A, Richardson SJ Nelson JF. Accelerated disappearance of ovarian follicles in mid-life - implications for forecasting menopause. Hum Reprod. 1992;7:1342-6.

9 Ferraretti AP, Goossens V, Kupka M, Bhattacharya S, de Mouzon J, Castilla JA, et al. Assisted reproductive technology in Europe, 2009: results generated from European registers by ESHRE. Hum. Reprod. 2013;28:1-14

10 Tarlatzis BC, Pados G. Oocyte donation: clinical and practical aspects. Mol Cell Endocrinol. 2000;161:99-102.

11 Budak E, Garrid N, Reis Soares S, Barreto Melo MA, Meseguer M, et al. Evolution of a large oocyte donation program over a 10-year period. Fertil Steril. 2007;88: 342-9.

12 Stoop D, Baumgarten M, Haentjens P, Polyzos NP, De Vos M, Verheyen G, Camus M, Devroey P. Reproductive Biology and Endocrinology. 2012;10:42.

13 De Geyter C. Assisted reproductive medicine in Switzerland. Swiss Med Wkly. 2012;142:w13569. 